\title{
Phyto- und Mykotoxine (5)
}

Yohimbin und andere pflanzliche Aphrodisiaka

Jean-Michel Jeannin

\section{Einleitung}

Die Steigerung der Libido und der Potenz scheinen ein sehr altes menschliches Bedürfnis zu sein. Nachfolgend werden hier aus einer eindrücklichen Fülle von pflanzlichen Präparaten und Lebensmitteln die wissenschaftlich interessantesten Heilpflanzen sowie zur Auflockerung einige amüsante Anwendungen von Lebensmitteln vorgestellt.

\section{Pausinystalia yohimbe Pierre ex Beille (Yohimbebaum)}

P. yohimba ist ein bis zu $30 \mathrm{~m}$ hoher immergrüner Urwaldbaum aus der Familie der Rubiaceae, mit ganzrandigen Blättern mit einem spitzen Ende und weissen oder gelben röhrenförmigen Blüten. Der Baum ist in West- und Zentralafrika heimisch. Arzneilich verwendet wird die Rinde bzw. ein Rindenextrakt [1].

\section{Chemie und Toxizität}

Die Rinde des Yohimbebaums enthält verschiedene Alkaloide, hierunter Yohimbin (ca. 6\% der Rohmasse), seine Derivate Yohimbinin, $\alpha$-Yohimbane, Yohimbenin usw. [2]. Der Yohimbebaum gehört zur Giftklasse 1b (sehr giftig) (Tab. 1). Allerdings sind bisher keine Todesfälle nach akuter oraler Überdosierung bekannt

Tab. 1. Giftklassen der WHO (nach [1])

\begin{tabular}{lll}
\hline Klasse & Bezeichnung & LD50 Ratte, mg/kg KG \\
\hline Ia & äusserst giftig & $\leq 5$ \\
Ib & sehr giftig & $5-50$ \\
II & giftig & $50-500$ \\
III & schwach giftig & $>500$ \\
\hline
\end{tabular}

\section{KARGER}

Fax +497614520714 Information@Karger.com www.karger.com

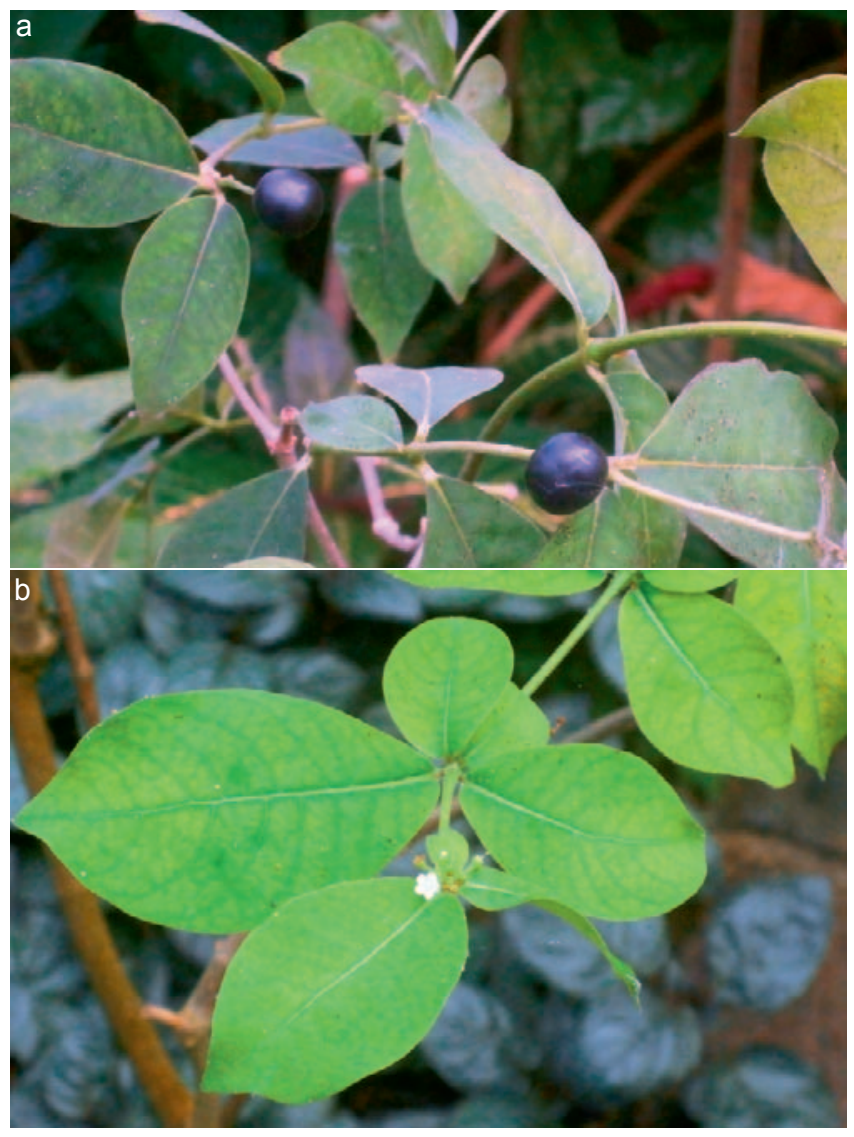

Abb. 1.a,b. Rauvolfia species. Botanischer Garten, Zürich, 01.09.2014.

geworden. Eine Dosis von 1,8 g (das 100-Fache einer üblichen Tagesdosis) führte zu mehrstündiger Bewusstlosigkeit; die komplette Genesung benötigte mehrere Tage. Die LD 50 bei der Maus für eine intravenöse Injektion beträgt $20 \mathrm{mg} / \mathrm{kg}$ [1]. Nach Frohe [3] gelten eine Einzeldosis von $18 \mathrm{mg}$ sowie eine Tagesdosis von $100 \mathrm{mg}$ als toxisch. Solche Dosen verursachen eine Schädigung des Herzens. 
Abb. 2. Yohimbin.

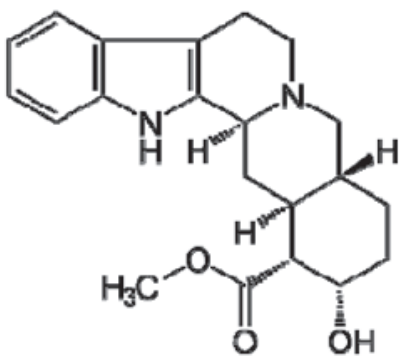

Abb. 3. $\alpha$-Yohimbin (Rauwolscin).

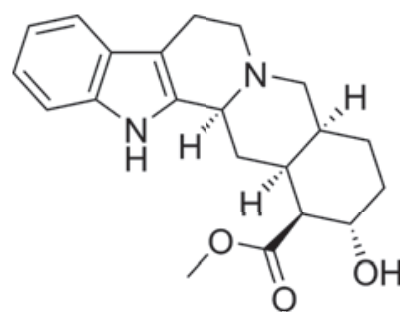

Rauvolfia serpentina L. (Rauwolfia; Schlangenholz), Rauvolfia ssp.

R. serpentina (Abb. 1) ist ein immergrüner, ca. 0,5-1,0 $\mathrm{m}$ hoher Strauch aus der Familie der Apocynaceae (Hundsgiftgewächse). Die glänzenden Blätter sind spitzeiförmig. Die Blüten sind weiss oder rosarot. Die erbsengrossen Steinfrüchte sind im reifen Zustand schwarz [1, 4]. Die Pflanze ist in Südostasien weit verbreitet [1].

\section{Chemie und Toxizität}

Die medizinisch wirksamen Inhaltsstoffe sind Alkoloide (in der Wurzel insgesamt 1-3\%), darunter Ajmalicin, Ajmalin, Deseripin, Reserpin, Rescinnamin (Reserpinin), Serpentin und Yohimbin $[1,4]$. Reserpin und Reserpinin sind Blutdrucksenker. Rauwolfia gehört zur Giftklasse 1b (sehr giftig). In Tansania ereignen sich jährlich 70 tödliche Vergiftungen mit Rauvolfia caffra [1]. Die in Afrika heimische Art Rauvolfia vomitoria enthält neben den zuvor beschriebenen Substanzen das YohimbinStereoisomer $\alpha$-Yohimbin [5], mit einer Yohimbin vergleichbaren Wirksamkeit. Aus der Wurzel von R. serpentina wurden die ersten modernen Antihypertonika entwickelt (das Thema soll in einem folgenden Beitrag behandelt werden).

\section{Pharmakologie und Toxikologie von Yohimbin und $\alpha$-Yohimbin}

Yohimbin (Abb. 2) greift mehrfach in das symphatische Nervensystem ein: Es ist ein $\alpha_{2}$-AdrenorezeptorAntagonist mit hoher Affinität und ein $\alpha_{1}$-Adrenorezeptor-Antagonist mit einer mässig hohen Affinität. Yohimbin ist mit teils mässig hoher, teils niedriger Affinität ein
Antagonist der 5HT(Serotonin)-Rezeptoren. Yohimbin und $\alpha$-Yohimbin (Abb. 3) sind sowohl zentral als auch peripher starke kompetitive Antagonisten des Serotonins, wobei das $\alpha$-Yohimbin wirksamer ist als das Yohimbin [5]. Der dritte Angriffspunkt sind die Dopamin-Rezeptoren $\mathrm{D}_{2}$ und $\mathrm{D}_{3}$, zu denen Yohimbin ein Antagonist mit mässig hoher und niedriger Affinität ist [6]. Die verschiedenen Affinitäten erklären, warum verschiedene Konzentrationen bzw. Dosierungen gegensätzliche Wirkungen auslösen: Nach niedrigeren Dosen löst die Blockierung der $\alpha_{2}$-Rezeptoren eine Blutdrucksenkung aus - im Gegensatz zu einer hohen Dosierung, die durch Blockierung der $\alpha_{1}$-Rezeptoren zu einer Blutdrucksteigerung führt [7]. Yohimbin gilt als Inhibitor von Monoaminooxidasen [7] und übt demnach eine antidepressive Wirkung aus. Bestimmte EEG-Veränderungen lassen eine anxiolytische Wirkung vermuten [8]. Yohimbin fördert die Lipolyse [8].

Als Hydrochlorid peroral verabreichtes Yohimbin wird rasch resorbiert $(<1 \mathrm{~h})$. Die Substanz wird in der Leber zum Hauptmetaboliten 11-OH-Yohimbin und zu einem Nebenmetaboliten 10-OH-Yohimbin hydroxyliert. Beide Metaboliten sind aktiv. 11-OH-Yohimbin ist in höherer Konzentration im Plasma nachweisbar. Die Eliminationshalbwertszeit beträgt für Yohimbin 0,92 h und für 11-OHYohimbin $6 \mathrm{~h}$, was die Diskrepanz zwischen den pharmakokinetischen Daten und der Wirkungsdauer erklärt. Beide Metaboliten werden im Urin ausgeschieden [8].

\section{Anwendung}

Yohimbin und seine Stereoisomere gelten als wertvolleWerkzeugefürdieErforschungvon $\alpha$-Adrenorezeptoren [5]. Die klinische Anwendung von Yohimbin ist Teil einer lebhaften Kontroverse [1]. Ernst und Pittler [9] untersuchten in einer Meta-Analyse von sieben randomisierten, Placebo-gesteuerten klinischen Studien die Wirksamkeit von Yohimbin zur Behandlung der erektilen Dysfunktion. Yohimbin war statistisch dem Placebo signifikant überlegen. Schwere unerwünschte Wirkungen waren selten und reversibel. Andere Studien zeigten nur teilweise eine Wirksamkeit von Yohimbin [8]. Da die erektile Dysfunktion auch ein sehr persönliches Problem bedeutet, dürften Ergebnisse von therapeutischen Studien schwer zu standardisieren sein; die Ergebnisse sind daher mit Vorsicht $\mathrm{zu}$ interpretieren. Wiewohl Yohimbin ein MAO(Monoaminooxidase)-Hemmer ist und diese nicht mit selektiven Serotonin-Wiederaufnahmehemmern (SSRI) kombiniert werden sollten, vermag die Substanz die unerwünschten Wirkungen letzterer auf die Sexualfunktion (Erektionsschwäche, Impotenz) abzuschwächen $[8,10]$. Eine Behandlung mit Yohimbin zur Steigerung des sexuellen Begehrens war in einer Studie an 11 Frauen 
Abb. 4. Spanischer Pfeffer (Capsicum annuum) oder Paprika.

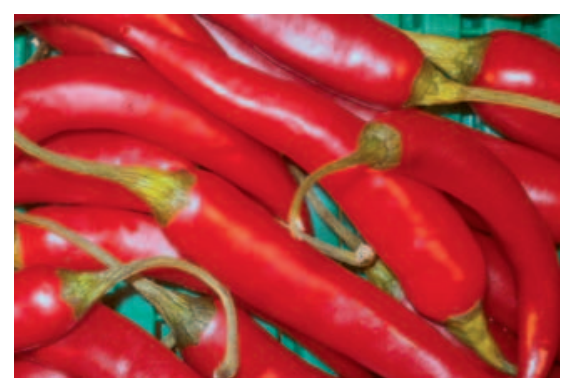

erfolglos [8]. Yohimbin fördert die Lipolyse nach akuter Verabreichung, nicht aber nach 14-tägiger Behandlung $(2 \times 4 \mathrm{mg} / \mathrm{Tag})$. Deshalb eignet es sich nicht als Behandlung zur Gewichtsreduktion [8]. Yohimbin wurde nach einer Überdosierung von Clonidin mit Erfolg als Antidot $(5,4 \mathrm{mg})$ verabreicht [8]. Yohimbin vermag möglicherweise einer Opiatabhängigkeit vorzubeugen [8]. Patienten, die an orthostatischer Hypotonie leiden, können möglicherweise von einer Behandlung mit Yohimbin profitieren [8].

Ein 42-jähriger depressiver, drogenabhängiger Patient unter antiretroviraler Therapie erlitt einen Priapismus nach Einnahme eines frei verkäuflichen YohimbinRindenpräparats, der mit einem chirurgischen Eingriff (Shunt) behandelt werden musste [11].
Abb. 5. Ingwer (Zingiber officinale).

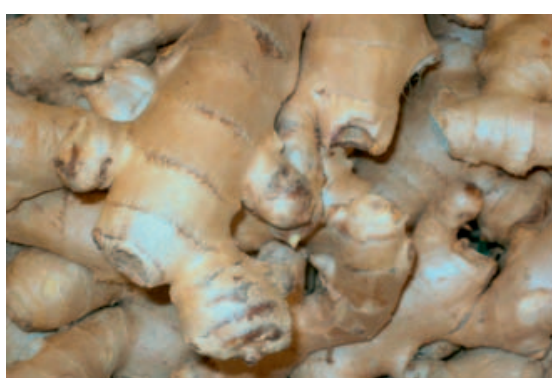

\section{Aphrodisiaka aus der Küche}

Die Gemüse Fenchel, Paprika (Capsicum annuum) (Abb. 4) und Sellerie, die Früchte Erdbeere und Granatapfel sowie die Gewürze Basilikum, Ingwer (Abb. 5), Muskatnuss (Myristica fragrans), Petersilie und Pfeffer sind in vielen Kochportalen die am häufigsten genannten Aphrodisiaka. Übereinstimmend wird angeraten, Paprika und Muskatnuss nur in mässigen Mengen einzunehmen. Sowohl Paprika als auch Muskatnuss werden auch in der Phytotherapie angewendet. C. annuum wird als lokales Schmerzmittel verwendet. Übermässiger Konsum ist schädlich (Giftklasse III) [1]. Das ätherische Öl aus der Nuss und den Blüten von $M$. fragrans wirkt spasmolytisch. Es gilt als Neurotoxin, führt zu Räuschen und kann abhängig machen (Giftklasse II) [1].

\section{Literatur}

1 Wink M, et al: Handbuch der giftigen und psychoaktiven Pflanzen. Stuttgart, WVG, 2008.

2 Leung AY, Forster S: Encyclopedia of Common Natural Ingredients, ed 2. New York, Wiley, 1996.

3 Frohe D: Pausinystalia johimbe (K. Schum.) Pierre ex Beille, Yohimbe; in Frohe D: Heilpflanzenlexikon, ed 8. Stuttgart, Wissenschaftliche Verlagsgesellschaft mbH, 2006.

4 IndischeSchlangenwurzel.http://de.wikipedia. org/wiki/Indische_Schlangenwurzel.
5 Apocynaceae; in Neuwinger HD: Afrikanische Arzneipflanzen und Jagdgifte, ed 2. Stuttgart, Wissenschaftliche Verlagsgesellschaft, 1998.

6 Millan M, et al: Agonist and antagonist actions of yohimbine as compared to fluparoxan at alpha 2 -adrenergic receptors (AR)s, serotonin $(5-\mathrm{HT}) 1 \mathrm{~A}, 5-\mathrm{HT} 1 \mathrm{~B}, 5-\mathrm{HT} 1 \mathrm{D}$ and dopamine D2 and D3 receptors. Significance for the modulation of frontocortical monoaminergic transmission and depressive states. Synapse 2000;35:79-95.

7 Yohimbine. http://en.wikipedia.org/wiki/ Yohimbine.
Tam S, et al: Yohimbine: a clinical review. Pharmacol Ther 2001;9:215-243.

9 Ernst E, Pittler MH: Yohimbine for erectile dysfunction: a systematic review and metaanalysis of randomized clinical trials. J Urol 1998;159:433-436.

10 Fecik S: Drug-induced sexual dysfunction. Medical Update for Psychiatrists 1998;3: 176-181.

11 Myers A, et al: Refractory priapism associated with ingestion of yohimbe extract. J Med Toxicol 2009;5:223-225. 